\title{
Complex Network Model for Characterizing Hazards and Risks Associated with Mine-tailings Facility
}

\author{
Shuang Gao ${ }^{1}$, Zhixin Zhen ${ }^{1}$, Zhongxue $\mathrm{Li}^{1}$, Yiqing Zhao ${ }^{1 *}$ and Xuan Qin ${ }^{2}$ \\ ${ }^{1}$ Department of Mineral Resources Engineering, University of Science and Technology Beijing, Beijing, China \\ ${ }^{2}$ Mine Safety Technology Research Institute, China Academy of Safety Science and Technology, Beijing, China
}

\begin{abstract}
If not well-managed, a mine-tailings facility may become a major source of risks, endangering the community and environment, and damaging the reputation of the minerals industry regarding sustainability. Identifying, characterizing, and mitigating the hazards and risks associated with tailings facilities have been critical to the maintenance of community-safe and environmentally sound mine-tailings facilities. Herein, a complex network model for characterizing the hazards and risks associated with the lifecycle of tailings facilities is presented. In this approach, the hazards are modeled as vertices of the complex network, and the interactions among the hazards are modeled as edges of the complex network. The complex network for modeling the hazard and risk spreading of mine-tailings impoundments is analyzed and characterized by using network metrics such as the network density, geometrical characteristics, characteristic path length, network efficiency, and clustering coefficient. The degree distribution of the network obeys a power-law distribution, indicating that the network for characterizing the risk spreading associated with a tailings facility is scale-free. According to the results of calculations and existing research results, the network is ultrasmall-world. By analyzing the change of the global network efficiency under four kinds of different methods to remove network nodes and edges, network nodes with higher between centrality (BC) are identified as critical. The removal of those critical nodes helps mitigate risks associated with a tailings facility and reveals the vulnerabilities to $\mathrm{BC}$ attacks.
\end{abstract}

Keywords: tailing impoundment, sustainable development, complex network model, scale-free property, ultrasmall world, vulnerability, between centrality

\section{Introduction}

The safety management of tailings impoundments is very important because it is crucial for the sustainable development of the minerals industry. If these facilities are not well-managed, they will threaten the ecological environment and communities under the sustained action of natural stress and human activities. Studies on tailing impoundments in foreign countries have involved their hazards, risks, and effects on the ecological environment (Nejeschlebová et al 2015, Duque et al 2015, Dinis and Fiúza 2013, Zhang 2013). In China, most of the research on accidents and disasters involving tailings impoundments are focused on the stability of dams, safety monitoring and evaluation, etc. (Zhang 2013, Li et al 2012, Peng et al 2012, Hu et al 2011, Wang et al 2012, Li et al 2010). For a long time, studies on tailings accidents focused on the analysis of a single tailings dam accident; the knowledge and means for characterizing the long-term dynamic evolution of the hazards and risks of tailing impoundments are lacking. The complex network can be used to analyze the interaction and evolutionary process of complex factors, and it has been used in studies focusing on centrality, the small world, the scale-free property, and robustness and vulnerability.
Recently, researchers have applied it to a wide range of scientific and engineering fields, such as the analysis of the spread of computer viruses, the spread and diffusion of information, transportation and aviation networks, power networks, supply chain networks (Yang and Zhang 2013, Dai 2012), disease prevention and control (Wang 2011), and the spread of natural disasters (Weng et al 2007).

On the basis of previous studies on the state and identification of hazards for tailings impoundments, in this study, we attempt to introduce the complex-network method for analyzing the effect and the evolutionary relationship between the hazards, accidents, and consequences of tailings impoundments. We used the Pajek software to analyze the characteristic parameters of the whole network and the subnet, together with simulations, to determine the statistical characteristics of the network. Finally, by using different methods to remove nodes of the network, the critical nodes were identified.

\section{Hazard Modeling Using a Complex Network}

First, through investigation and analysis for tailings impoundments and related accidents at home and abroad, combined with the relevant procedures, norms, standards,

* Corresponding Author: Y.Q. Zhao, zyq@ustb.edu.cn, phone: +86 18500225299

Copyright @ 2017 Canamaple Academia Services, http://press.camdemia.ca

DOI: $10.15273 /$ gree.2017.02.019 
guidelines, cases, the hazards and accident risks are systematically identified according to the main stages of the lifecycle for tailing impoundments - including site selection, construction, operation, and closing tailings impoundments - considering factors such as the technology, man-made factors, the environment, and management. The nodes of the complex network represent the hidden dangers of technology, the unsafe behavior of people, the

Occurrence of earthquake with exceeding design intensity

Phusical properties of talilings (relative density, particle size, etc.)

DN and windv climate $\backslash$

Landside in impoundment area

Heaw raintall of exceedina the standard.

Loess area with larae spacino and vertical ioint

QPasses and avens caused bu cave development in karst area

Not timelv facility maintenance

Faultiness of rules and reaulations

Farmers' illecal behaviors includino sand exaction explosive fishing etc.

Flood-carrvina sustem without renovation betore closina a tallinas imooundmen

L Lack of renovation to a talilinas dam betore closina a talinas imboundment

QTalinas barren rock.waste water and darbace outside design plans into a talilings impoundment Seismic liquelaction of dam body

Animals nidilicale and burrow in the dam body

Lack of personnel's qualification and experience -

4.ack of storage capacity for flood regulation

Faultiness of security contro o Damage and tallure of drainage installation

Inadequate satety evaluation

Waste water discharge of exceeding standards s. Damage and failure of flood-carming tacilities

Irrational design of fllood discharge lacilities s Soaking delormation

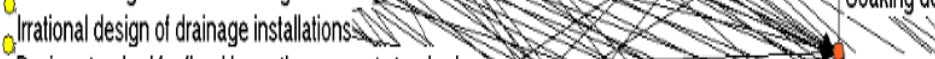

Design standard for llood lower than current standards $\Rightarrow$ - S C C acks of dam body

Irrational design for dam body structure and section size

Sand excavation without regulations and plans in closing talilings impoundments

Destruction of plants on dam slope 3 .

Irrational dam foundation treatment during damming 3

Uneven dam body density ${ }^{2} \rightarrow$ Scouring dam body

Dam materials not salistying the requirements $\bar{x}$

Irrational construction of inverted filter $\geq *$

Poor construction of fllood discharae facilities

Poor construction of drainage installations - -

(rising water level

Unreliable monitorina facilites

Excessivellyhigh primary dam

Oversteepened slope of fill dam $2 \longrightarrow$ Lack of llood-carrying capacity

Inadequate quantities of the opening branch pipe used to draw ore
Uneven ore drawing- - Minimum dry tallings beach length and tree board not satistying the design requirements

Sinde workina area to draw ore for a long time

Long-term unchanged drawpoint

Leakage of pipe fitings used as ore drawing

Excessive pursuit of bachwater quality

Pipeline break for transporting talilings

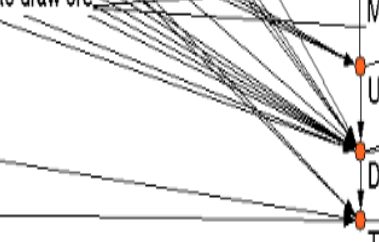

Talings leakage

Seepage tailure

Figure 1. Complex network for charactering the hazard and risk spreading associated with a mine-tailings impoundment.

The complex network model of dynamic risks for tailings impoundments consists of 65 hazardous nodes and 228 edges. The yellow-node layer on the left side represents the initial dormant hazards, including the hidden dangers of technology, unsafe human behavior, adverse environmental conditions, and lack of regulatory standard operating procedures such as unreliable monitoring facilities, heavy rainfall, and inadequate safety evaluation. The middle orange-node layer represents armed hazards formed by the impact factors or initial dormant hazards and may lead to accidents in some working environments or conditions, such as excessively high seepage lines, a lack of storage capacity 
for flood regulation, or the damage or failure of floodingcarrying facilities. These armed hazards indicate that accidents and disasters are imminent. The red-node layer on the right side represents the final active hazards. If these active hazards cannot be effectively controlled, serious consequences and accidents may occur.

\section{Network Characteristics}

The complex network has a series of structural characteristics. Thus, analysis methods and the structural characteristic parameters of the complex network can be used to analyze and express some of the characteristics and the regularity of the complex network for characterizing the risks associated with a tailings impoundment.

\subsection{Characteristic parameters of the network}

Taking the complex network of a tailings impoundment as an example, the final accidents or hazards on the right side of Figure 1 include five categories: floods overtopping, slump or collapse, seepage damage, dam break, and environmental pollution. To further analyze the characteristics of the complex network, these final hazards or accidents, dormant hazards, and armed hazards are divided into five sub-networks, and then characteristic parameters are obtained using the Pajek software. The relevant parameter values for the whole network and the five accident subnets are shown in Table 1. Via further analysis, additional information can be gained, as follows.

\section{(1) Network density}

The network density refers to the ratio of the actual number and the theoretical maximum of edges in the network, indicating the degree of closeness between the hazards. The results in Table 1 show that the number of hazardous nodes and the number of edges in the five accident subnets are smaller than the number of hazardous nodes and the number of edges of the whole network, respectively. However, the sum of the nodes and the sum of the edges are greater than the number of dangerous nodes and the number of dangerous edges in the whole network, respectively, which can mean that the whole network is not simply the sum of all the subnets and that a hazard may evolve into multiple types of accidents. The network density of the whole network and the accident sub-network for tailings impoundments is not more than $8 \%$, which indicates that the overall network density is low and that the influence factors, hazards, and path of risk spreading are single and unique.

Table 1. Values of characteristic parameters for characterizing dynamic risks of the whole network and subnets.

\begin{tabular}{ccccccc}
\hline Name of parameters & $\begin{array}{c}\text { Whole network of } \\
\text { risks of tailings } \\
\text { impoundments }\end{array}$ & $\begin{array}{c}\text { Subnet of } \\
\text { accidents of } \\
\text { floods } \\
\text { overtopping }\end{array}$ & $\begin{array}{c}\text { Subnet of } \\
\text { accidents of } \\
\text { slump or } \\
\text { collapse }\end{array}$ & $\begin{array}{c}\text { Subnet of } \\
\text { accidents of } \\
\text { seepage failure }\end{array}$ & $\begin{array}{c}\text { Subnet of } \\
\text { break-dam } \\
\text { accidents }\end{array}$ & $\begin{array}{c}\text { Subnet of } \\
\text { accidents of } \\
\text { environmental } \\
\text { pollution }\end{array}$ \\
\hline Number of nodes & 65 & 29 & 47 & 35 & 52 & 27 \\
$\begin{array}{c}\text { Number of edges } \\
\text { Network density }\end{array}$ & 228 & 65 & 122 & 70 & 134 & 32 \\
$\begin{array}{c}\text { Average degree of } \\
\text { network }\end{array}$ & 7.02 & $4 \%$ & $6 \%$ & $6 \%$ & $5 \%$ & $5 \%$ \\
$\begin{array}{c}\text { Degree centralization } \\
\text { (out/in) }\end{array}$ & $10 \% / 38 \%$ & $10 \% / 51 \%$ & $10 \% / 56 \%$ & $9 \% / 54 \%$ & $11 \% / 51 \%$ & $3 \% / 39 \%$ \\
$\begin{array}{c}\text { Characteristic path } \\
\text { length }\end{array}$ & 1.61 & 1.52 & 1.44 & 1.35 & 1.55 & 1.46 \\
$\begin{array}{c}\text { Network diameter } \\
\text { Global network } \\
\text { efficiency }\end{array}$ & 3 & 3 & 3 & 3 & 3 & 2.37 \\
Clustering coefficient & 0.08 & 0.11 & 0.07 & 0.07 & 0.07 & 0.06 \\
\hline
\end{tabular}

(2) Average degree of network and the degree centralization

The degree of a node in a network refers to the number of other nodes directly connected to the node-including the in-degree and out-degree - and indicates the direct influence of the node. The average degree of a network is the average value of all the nodes in the network and can reflect the average level of the direct influence of the nodes. The degree centralization is used to measure the degree of concentration of the network for one or some special nodes and reflects the concentration trend of the degree of the nodes in the whole network. The average degree of the network of risks spreading for tailings impoundments is
7.02, which indicates that there is a direct causal relationship between a hazard and the average of the seven hazards in the whole network. For subnets, the average degree of the slump/collapse and the break dam is larger than that for the other three subnets, which indicates that these two subnets have more hazards. The in-degree centralization is larger than the out-degree centralization in the whole network and the subnets, which suggests that the number of effect factors and hazards in tailingsimpoundment accidents is large but that of accidents is small.

(3) Characteristic path length of the network and network diameter 
The characteristic path length of the network represents the average value of the shortest path length between all the pairs of nodes in the network. It reflects the average distance of all the nodes in the network. The network diameter refers to the maximum distance between all the pairs of nodes in the network (Watts 1999). The characteristic path length of the network of risks spreading for tailings impoundments is 1.61, which indicates that a hazardous node affects other nodes after passing 1.61 network edges. The characteristic path lengths for the five subnets are smaller than that of the whole network, which indicates that the average distance between the hazards in the subnets is smaller than that in the whole network and that it is very easy to trigger the accident. The diameters of the five subnets and the whole network are all less than or equal to 3 , which shows that a hazard can reach the final activity hazards and lead to a tailings impoundment accident after three steps.

\section{(4) Global network efficiency}

The global network efficiency refers to the average value of the sum of the reciprocal of the shortest path lengths between all the pairs of nodes in the whole network and reflects the propagation speed of matter, information, or energy in the network (Latora and Marchiori 2003, Latora and Marchiori 2001). The global network efficiency of the network of risks spreading for tailings impoundments is 0.08 .

\section{(5) Clustering coefficient}

The clustering coefficient refers to the proportion of all nodes around a node that are interconnecting and can reflect the clustered condition of nodes in the local network (Watts and Strogatz 1998a). The clustering coefficient of the whole network is 0.21 , and the coefficients of five subnets are between 0.17 and 0.32 . In contrast, the clustering coefficient of a random network of the same scale is approximately 0.05 , which indicates that the whole network and the five subnets have a large degree of clustering.

\subsection{Characteristic analysis of the network}

\section{(1) Scale-free property}

The complex network is called the scale-free network if its degree distribution obeys the power-law distribution (Almaas and Barabási 2004, Barabási and Albert 2000). We randomly detect a node $x$ in a network. When the degree of the node is exactly $k, p(k)$ denotes the probability of the degree of the node. If the relationship between $p(k)$ and $k$ can be expressed by the power function $p(k)=k^{-\gamma}$, the degree of this network is subject to the power-law distribution, and the power exponent is $\gamma$. In practical applications, we first need to obtain the relationship between $p(k)$ and $k$. Then, the logarithmic operations are performed on both sides of the equation, and we can obtain the linear relationship between $l n^{p(k)}$ and $l n^{k}$. If the linear expression can be represented by a straight line with a negative exponent, the degree value of the network obeys the power-law distribution.

After statistical calculation, the degree distribution of the complex network for characterizing the hazards and risks associated with a mine-tailings facility is shown in
Figure 2. We can obtain the relational expression between the degree value and the probability:

$$
p(k)=0.1932 k^{-0.807}
$$

Both sides of formula (1) take logarithms, and we can obtain the relational Figure 3 using $\log$-log coordinates and the following linear expression:

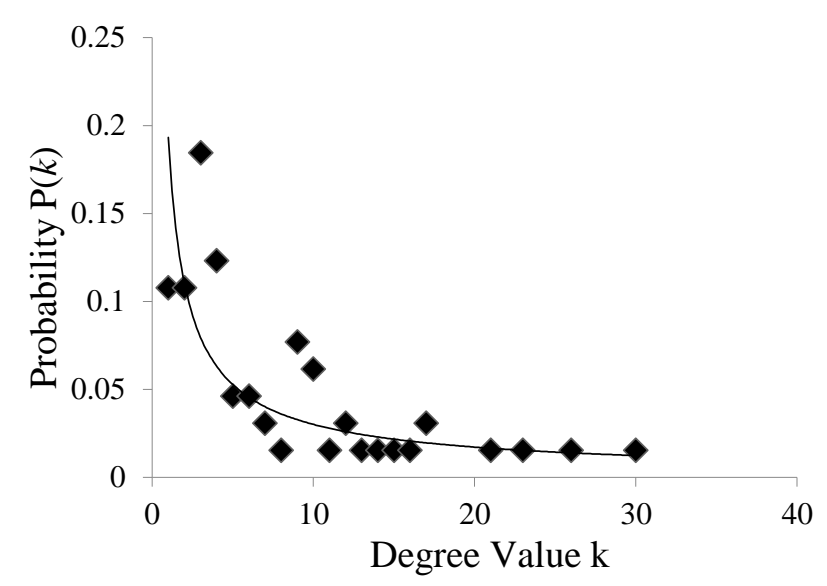

Figure 2. Degree distribution of the complex network.

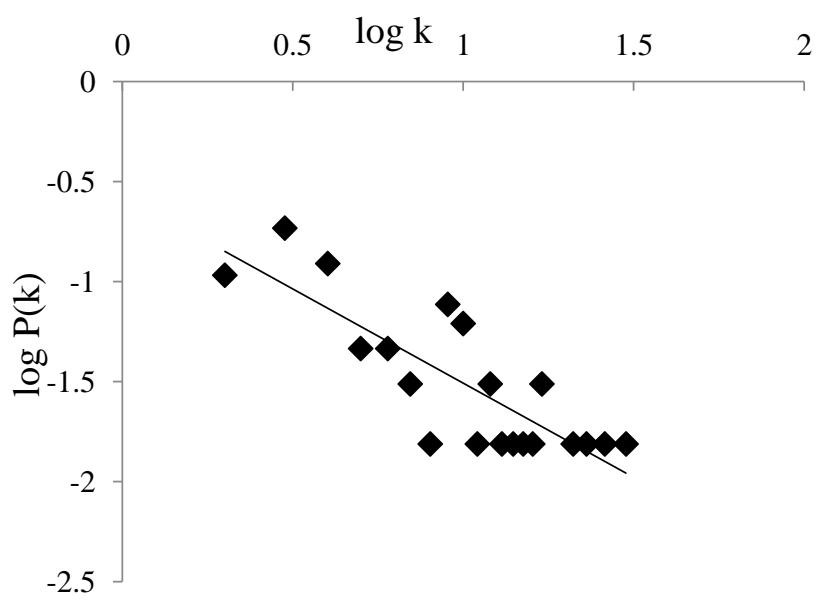

Figure 3 Degree distribution of the complex network using log-log coordinates.

$$
\ln ^{p(k)}=-0.9419 \ln ^{k}-0.5657
$$

The results show that the degree distribution obeys the power-law distribution; thus, the complex network for characterizing the hazards and risks associated with a tailings impoundment is a scale-free network.

\section{(2) Property of the ultrasmall world}

Watts and Strogatz (1998b) and Strogatz (2001) considered that the small-world network falls between a regular network and a random network. Later, physicists used the clustering coefficients and the characteristic path length to identify whether a network has the small-world property. The clustering coefficient and the characteristic path length of an ideal small-world network are approximately those of the corresponding regular network 
and random network, respectively (Watts and Strogatz 1998c, Huang 2011a).

If a random network has same the number of nodes and average degree value as the complex network that is used to characterize the risks spreading in a mine-tailings impoundment, the characteristic path length and the clustering coefficients of the random network can be expressed by formula (3). Similarly, these two statistical characteristics of the regular network are given by formula (4).

$$
\begin{aligned}
& \left\{\begin{array}{l}
L_{\text {Random }}=\ln ^{n} / \ln ^{k} \\
C_{\text {Random }}=k / n
\end{array}\right. \\
& \left\{\begin{array}{l}
L_{\text {Regular }}=(n(n+k-2)) /(2 k(n-1)) \\
C_{\text {Re gular }}=(3 k-6) /(4 k-4)
\end{array}\right.
\end{aligned}
$$

Here, $k$ represents the average degree value of a network, and the $n$ is the number of nodes. $L$ and $C$ are the characteristic path length and the clustering coefficient of the complex network, respectively. Through calculations, we obtain $L_{\text {Re gular }}=5.065>L_{\text {Random }}=2.1452>L=1.61 \quad$ and $C_{\text {Regular }}=0.625>C=0.21>C_{\text {Random }}=0.108$. According to the traditional expression for the small-world property, $L_{\text {Random }}$ should be greater than $L$; however, our result is different. Does this show that the complex network for characterizing the hazards and risks associated with a tailings impoundment lacks the small-world property?

According to related research (Cohen and Havlin 2003a), if a complex network has the free-scale property, its characteristic path length is smaller than that of the corresponding regular random network. This kind of network is ultrasmall-world. In addition, we can use the Euclidean metric to calculate the small-world measure $\delta$, which is defined as (Huang 2011b):

$$
\delta=1 /\left(1+\sqrt{\left(C-C_{\mathrm{Re} \text { gular }}\right)^{2}+\left(L-L_{\text {Random }}\right)^{2}}\right)
$$

The result is $\delta=0.5962$, which shows that the complex network for charactering the risks spreading associated with a tailings impoundment has a strong small-world property. Comparing the whole network of the risks spreading of a tailings impoundment with the subnets of accidents reveals that the characteristic path length of the whole network is greater than that of subnets, while the clustering coefficients of the subnets of accidents involving floods overtopping and seepage failure are larger than that of the whole network, and those of the other types of accidents are equal or smaller. These results show that the complex network used to characterize the risks in a mine-tailings impoundment has a partial small-world property, which verifies the foregoing evidence and calculation.

\section{(3) Centrality and the identification of critical nodes}

Regarding the centrality, we use quantitative methods for each node in the entire network to describe the extent of the center role, thereby finding the core of the whole network ( $\mathrm{Li}$ et al. 2012). That is, via the centrality analysis, we can determine the position or difference of the hazardous nodes in the network for characterizing the hazards and risks associated with a tailings impoundment. In this study, the centrality includes three aspects: degree centrality (DC), between centrality (BC), and closeness centrality (CC).

1) $\mathrm{DC}$

The DC is used to describe the direct influence of a node in a static network, which can reflect the ability to build direct connections between the node and its surrounding nodes. In a directed network, the degree of each node can be divided into the out-degree and in-degree. The in-degree of a node is the number of other nodes that point to the node, and the out-degree of a node is the number of connections directly made by the node.

For the nodes of the larger out-degree, man-made and management factors account for a large proportion, which can indicate that this kind of factor affects the risk spreading to a greater extent. Among these factors, the out-degree of 'occurrence of earthquake with exceeding design intensity' is largest. After the in-degrees of the nodes in the network are ranked, we discover that accidents involving 'slump and collapse' and 'seepage damage' are arranged in the forefront, indicating that many hazards can cause these accidents. 2) $\mathrm{BC}$

The BC reflects the effects of nodes and edges and is used to measure the control degree of a node for its surrounding edges and nodes. According to the definition and calculation of the $\mathrm{BC}$, the average $\mathrm{BC}$ of the network nodes is 0.0011 , the maximum value is 0.0099 , the minimum value is 0 , and the standard deviation is 0.0023 . These data show that the BC of the network of the risks spreading for tailings impoundments has very strong heterogeneity. The maximum value is nine times the mean value, and the standard deviation is twice the mean value, which indicates that there are a small number of nodes with a large $\mathrm{BC}$ in the network.

3) $\mathrm{CC}$

The CC is an important indicator for centrality analysis and is determined by measuring the closeness or distance between the nodes in the network. If all the distances between a node and the other nodes in the network are small, the node has a higher CC. For directed networks, the distance is measured according to the edge of the same direction; thus, to determine the $\mathrm{CC}$ of the directed network, the in-closeness and out-closeness must be calculated separately. The maximum out-CC of the network is 0.1962 , the minimum value is 0 , the mean is 0.0799 , and the standard deviation is 0.0443 . For the in-CC, the maximum is 0.5372 , the minimum is 0 , the mean is 0.0746 , and the standard deviation is 0.1385 . The mean values for the network indicate that both the in-closeness and outcloseness are very small. The standard deviation of the incloseness is 3 times that of the out-closeness. Clearly, the out-closeness is more concentrated, which can indicate that the armed hazards and eventually active hazards become more concentrated after the hazard states change.

4) Identification of critical nodes

The three aforementioned methods are used to identify the core nodes (hazards). However, for the formation and spreading of risks in a mine-tailings facility, we cannot determine which method can identify the critical nodes 
having the greatest impact.

To identify these critical nodes, we can observe the change of the global network efficiency by removing the nodes with a high DC, high CC, and high BC, as well as random nodes in the network.

For ensuring a high accuracy, the simulation of removing random nodes was performed 20 times to determine the mean of the global efficiency. The process of removing nodes and edges is known as a network attack (Holme et al 2002).

According to the results of Pajek, the nodes in the network were removed according to the descending order of values of $\mathrm{DC}, \mathrm{CC}$, and BC. Because the nodes of the finally active hazards in the network are the main research objects, we did not remove these hazardous nodes in the attack analysis. Figure 4 shows the change of the global network efficiency under different attack schemes.

According to Figure 3, the global network efficiency exhibits a decreasing trend under the four kinds of attack schemes. When the global network efficiency is 0 , there is no connection between nodes in the network, and risks spreading of the tailings impoundment are effectively controlled. When the proportion of removing nodes is the same under the four kinds of schemes, the global network efficiency of a BC attack decreases rapidly. When the global network efficiency decreases to the same level, the proportion of nodes removed by a BC attack is the smallest. It can be concluded that the critical nodes in the dynamic network for charactering the hazards and risks associated with a tailings impoundment are the nodes with a high BC. These hazardous nodes should be checked and controlled first.

In addition to the aforementioned results, comparing the change of the global network efficiency under a random attack with that under other attack schemes, reveals that the complex network has strong robustness under random attacks but is vulnerable to selective attacks of nodes with a high BC. This conclusion is consistent with the properties of a free-scale network (Cohen and Havlin 2003b).

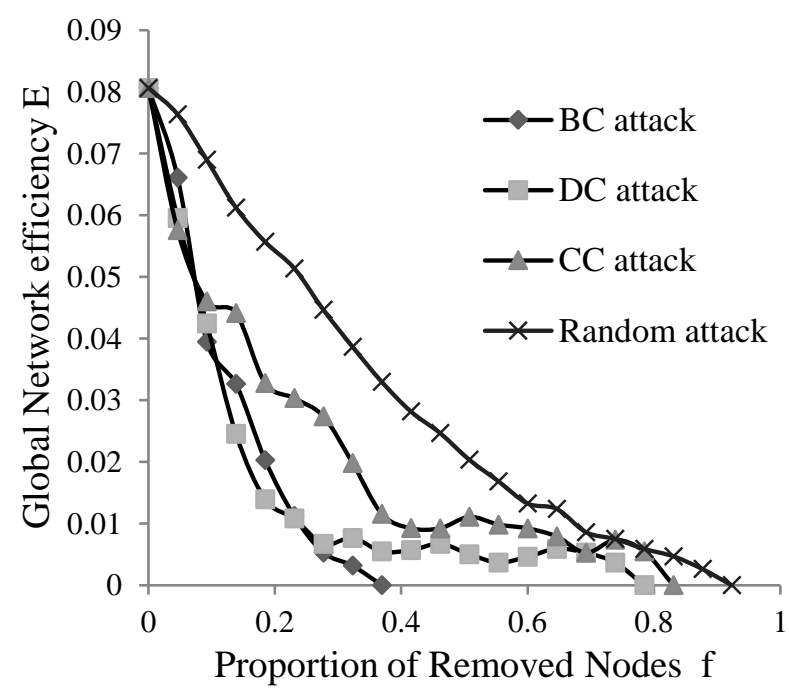

Figure 4. Variability of the global network efficiency under different attack schemes.

\section{Conclusions}

(1) According to hazard identification, a complex network for charactering the hazards and risks spreading associated with a mine-tailings facility is developed. The 65 nodes of the network are divided into three layers (factors, hazards, and accidents and consequences) or two stages (factors to hazards and hazards to accidents and consequences), which shows the spreading path of risks in a mine-tailings facility.

(2) We showed that in the complex network for charactering the risks spreading and hazards associated with a tailing facility, the relationship between each degree value and its probability under log-log coordinates could be represented by a straight line with a negative exponent, which means that the degree distribution of the complex network obeys the power-law distribution and that the network is free-scale.

(3) Comparing the relationship between the clustering coefficient and the characteristic path length for the complex network with a random network and regular network with the number of nodes and average degree value, combined with existing research results, reveals that the complex network has the ultrasmall-world property. When we calculate the small-world measure or compare the whole network with the subnet, we discover that the complex network is partially small-world.

(4) The rate of decrease of the global network efficiency is highest under a BC attack. That is, the nodes with a high BC are the critical nodes. Additionally, comparing the change of the global network efficiency under the random attack with that under other attack schemes reveals that the complex network has the robustness to the random attack and a vulnerability to the BC attack.

\section{Acknowledgement}

This research project is made possible through the financial support from the National Key Research and Development Program of China under Grant No. 2017YFC0804600.

\section{References}

Almaas, E. and A. Barabási, 2004. Power laws in biological networks. Arxiv Cornell University Library: 1-11.DIO: 10.1007/0-387-33916-7_1.

Barabási, A.L., R. Albert, H. Jeong, A.L. Barabási, R. Albert, and H. Jeong, 2000. Mean-field theory for scale-free random networks. Physica A Statistical Mechanics \& Its Applications, 272(1-2): 173 - 187.

Cohen, R. and S. Havlin, 2003. Scale-free networks are ultrasmall. Physical Review Letters, 90(5): 058701.

Dai, M.M., 2012. The research on evolution model and risk spread model of complex industrial chain networks. Master of Science Thesis, Nanjing University of Aeronautics and Astronautics, Nanjing.

Duque, J.F., M.I. Zapico, R. Oyarzun, J.A.L. García and P. Cubas. 2015. A descriptive and quantitative approach regarding erosion and development of landforms on abandoned mine tailings: New insights and 
environmental implications from SE Spain. Geomorphology, 239: 1 - 16.

Dinis, M.D.L. and A. Fiúza, 2013. Occupational exposure during remediation works at a uranium tailings pile. Journal of Environmental Radioactivity, 119(5): 63.

Holme, P., B.J. Kim, C.N. Yoon and S.K. Han, 2002. Attack vulnerability of complex networks. Physical Review E, 65(5): 056109.

Hu, W.Z., H.T. Ma, Z.H. Xie and Q.M. Li, 2011. Application of three dimensional high-density resistivity method in hidden danger detection of tailings. Journal of Safety Science and Technology, 7(9): 64 - 67.

Huang, X.J., 2011. Analysis of the small-world characteristics and stability of industrial symbiosis network evolution. Journal of Northeastern University, 32(6): 887 - 890 .

Latora, V. and M. Marchiori. Economic small-world behavior in weighted networks. The European Physical Journal B-Condensed Matter and Complex Systems, 2003, 32(2): 249 - 263.

Latora, V. and M. Marchiori, 2001. Efficient behavior of small-world networks. Physical Review Letters, 87(19): 198701.

Li, Z.Q., R.X. Zhang, Z. Yang, H.Z. Zhao and J.H. Yu, 2012. Influence complex network centrality on disaster spreading. Physics, 61(23): 514 - 518.

Li, Q., L.T. Zhang, Q.L. Qi and Z.L. Zhou. 2012. Instability characteristics and stability analysis of a tailings dam based on fluid-solid coupling theory. Rock and Soil Mechanics, 33(S2): 243 - 250.

Li, Z.X., Z.G. Cao and Y.Q. Zhao, 2010. Safety case and PDCA based safety assurance system for mine tailings facilities. Systems Engineering - Theory \& Practice, 30(5): 936 - 944.

Nejeschlebová, L., O. Sracek, M. Mihaljevič, V. Ettlerb, B. Kř́ibekc, I. Knéslc, A. Vaněkd, V. Penížekd, Z. Dolníčeka and B. Mapanie, 2015. Geochemistry and potential environmental impact of the mine tailings at
Rosh Pinah, southern Namibia. Journal of African Earth Sciences, 105: 17 - 28.

Peng, K., X.B. Li, S.M. Wang, G.Y. Zhao and Z.X. Liu, 2012. Optimization model of unascertained measurement for dam-break risk evaluation in tailings dams. Journal of Central South University (Science and Technology), 43(4): 1447 - 1452.

Strogatz, S.H., 2001. Exploring complex networks. Nature, 410(6825): 268.

Wang, Y.B., L. Wang and Z.X. Li, 2012. Safety evaluation of mine tailings facilities based on HS-BP algorithm. Systems Engineering - Theory \& Practice, 32(11): 2585 $-2590$.

Wang, Y.Q., 2011. Epidemic spreading on complex networks with multiple transmission factors and its immunization strategies. Ph.D. Thesis, Nanjing University of Posts and Telecommunications, Nanjing.

Watts, D.J. and S.H. Strogatz, 1998. Collective dynamics of 'small-world' networks. Nature, 393(6684): 440 - 442.

Watts, D.J., 1999. Small worlds: The dynamics of networks between order and randomness, Princeton university press, New Jersey, USA.

Weng, W.G., S.J. Ni, S.F. Shen and H.Y. Yuan, 2007. Dynamics of disaster spreading in complex networks. Acta Physica Sinica, 56(4): 1938 - 1943.

Yang, K. and Z.Y. Zhang, 2013. The research on mechanism of supply chain network risk based on complex network theory. Journal of Systems Science and Mathematical Sciences, 33(10): 1224 - 1232.

Zarroca, M., R. Linares, P.C. Velásquez-López, C. Roqué and R. Rodríguez, 2015. Application of electrical resistivity imaging (ERI) to a tailings dam project for artisanal and small-scale gold mining in ZarumaPortovelo, Ecuador. Journal of Applied Geophysics, 113: 103 - 113.

Zhang, L.T., 2013. Summary on the dam-break of tailing pond. Journal of Hydraulic Engineering, 44(5): 594 660. 